\title{
Study Subject Experience Document Version
}

National Cancer Institute

\section{Source}

National Cancer Institute. Study Subject Experience Document Version. NCI Thesaurus.

Code C153135.

A document capturing the actual end-to-end (or beginning to point-in-time) experience of a single study subject within the context of a particular study. 